\title{
TOPOLOGICAL SPACES WITH POINT-NETWORKS
}

\author{
ZOLTÁN BALOGH
}

\begin{abstract}
In this paper we introduce the notion of a pcint-network and prove that in $T_{1}$ spaces having a point-network, countable pseudocharacter implies stratifiable. As a corollary, there follows Reed's theorem that a $T_{1}$ space is metrizable if and only if it has an open point-network.
\end{abstract}

0. Introduction. The basic idea in this paper is that of a point-network: Let $X$ be a topological space, and suppose that to every point $x$ of $X$, we have assigned a decreasing chain $\{N(m, x): m \in \omega\}$ of subsets containing $x$. Then we call the system $\mathscr{N}=\{N(m, x): x \in X, m \in \omega\}$ a point-network for $X$ if, for every open subset $U$ of $X$ and every point $x$ of $U$, there is an set $V[x, U] \ni x$ such that whenever $y \in V[x, U]$ there is an $m(=m(y))$ with $x \in N(m, y) \subset U$. This idea is closely related to a conjecture of P. J. Collins and A. W. Roscoe $\left[\mathbf{C R}_{2}\right]$ that a $T_{1}$ space is metrizable if and only if it has a point-network such that each $N(m, x)$ is a neighbourhood of $x$. This conjecture, as they realized later, was wrong: McAuley's "bow-tie" space [Ce] gives a counterexample. Then they wondered whether spaces satisfying the conditions of their conjecture had at least $G_{\delta}$-diagonals $\left[\mathbf{C}_{2}\right]$, and whether assuming that every $N(m, x)$ is an open neighbourhood would be enough to imply metrizability $\left[\mathbf{C}_{\mathbf{1}}\right]$. The latter conjecture was first answered by G. M. Reed [R] who proved the beautiful theorem that a space is metrizable if and only if it has an open point-network. (At this point, the reader is invited to consult with [CR], where it is shown that even a weaker version of Reed's theorem implies all the known general metrization theorems.) An affirmative answer to the first question is due to the author and is the subject matter of the present paper. Actually, we prove the much stronger theorem that spaces with a point-network and countable pseudocharacter are stratifiable (Theorem 2.2). Having received Reed's notes [R] the present author realized that his theorem implies Reed's.

Throughout the paper we use standard notation and terminology of current set-theoretic topology. Stratifiable spaces are defined in $\$ 2$. When we use the term " $Y$ is a $\sigma$-discrete subspace" we mean that $Y$ is the union of countably many (not necessarily closed) discrete subspaces.

All other undefined notions can be found in [E], for example.

Received by the editors December 12, 1983 and, in revised form, April 2, 1984.

1980 Mathematics Subject Classification. Primary 54E20, 54E35; Secondary 54D18.

Key words and phrases. Point-network, collectionwise normal, stratifiable, metrizable. 
1. Covering properties and examples. Let $X$ be a space with a point-network $\{N(m, x): x \in X, m \in \omega\}$ and let us fix an operation $V[x, U]$ such as required in the definition of a point-network. Then a crucial observation is

Proposition 1.1. If $x, y \in X$ and $U, W$ are open sets with $x \in U \subset X-\{y\}$, $y \in W \subset X-\{x\}$, then $V[x, U] \cap V[y, W]=\varnothing$.

Proof. Suppose indirectly that there is point $z \in V[x, U] \cap V[y, W]$. Then there are $m, k \in w$ such that $x \in N(m, z) \subset U$ and $y \in N(k, z) \subset W$. Without loss of generality we may assume $m \leqslant k$. Then $y \in N(k, z) \subset N(m, z) \subset U \subset X-\{y\}$, a contradiction.

COROllaRY 1.2. Every $T_{1}$ space $X$ with a point-network is (hereditarily) collectionwise normal.

Proof. Let $\left\{F_{\alpha}: \alpha \in \lambda\right\}$ be a closed discrete collection in $X$. Then there is an open collection $\left\{O_{\alpha}: \alpha \in \lambda\right\}$ such that $F_{\alpha} \subset O_{\alpha} \subset X-\bigcup_{\beta \in \lambda-\{\alpha\}} F_{\beta}$ for every $\alpha \in \lambda$. Now, by Proposition 1.1, the collection $\left\{O_{\alpha}^{\prime}: \alpha \in \lambda\right\}$, where $O_{\alpha}^{\prime}=\bigcup\left\{V\left[x, O_{\alpha}\right]\right.$ : $\left.x \in F_{\alpha}\right\}$, is a simultaneous separation of the $F_{\alpha}$ 's by open sets.

Since "having a point-network" is clearly a property inherited by subspaces, this also proves that $X$ is hereditarily collectionwise normal.

LEMMA 1.3. Every $T_{1}$ space $X$ with a point-network is (hereditarily) paracompact.

PrOOF. Since "having a point-network" is a hereditary property, and collectionwise normal metacompact spaces are paracompact, we only have to prove that $X$ is metacompact. To see this, let $\mathcal{O}=\left\{O_{\alpha}: \alpha \in \lambda\right\}$ be an open cover of $X$, let $M_{\alpha}=O_{\alpha}-\cup_{\beta<\alpha} O_{\beta}(\alpha \in \lambda)$, and for every $x \in X$ let $o(x)$ denote the unique ordinal $\alpha \in \lambda$ with $x \in M_{\alpha}$. For every $\alpha \in \lambda$ let

$$
O_{\alpha}^{\prime}=\bigcup\left\{V\left[x, O_{\alpha}\right]: x \in M_{\alpha}\right\} \text {. }
$$

We claim that $\mathcal{O}^{\prime}=\left\{O_{\alpha}^{\prime}: \alpha \in \lambda\right\}$ is a point-finite open refinement of $\mathcal{O}$. Only "point-finite" needs proof. Suppose indirectly that there is an $x \in X$ such that $x$ is in infinitely many members of $\mathcal{O}^{\prime}$. Let $m \in \omega$ be the smallest with $N(m, x) \subset O_{o(x)}$. Clearly, there is a sequence $o(x)<\alpha_{0}<\cdots<\alpha_{m}$ of ordinals such that $x \in O_{\alpha_{k}}^{\prime}$ $(k=0, \ldots, m)$. By (1) this implies that there are points $y_{k} \in M_{\alpha_{k}}(k=0, \ldots, m)$ each having the property $x \in V\left[y_{k}, O_{\alpha_{k}}\right]$. It follows that there are $i_{k} \in \omega(k=0, \ldots, m)$ with $y_{k} \in N\left(i_{k}, x\right) \subset O_{\alpha_{k}}$. Let us consider now any pair of indices $k, l$ with $k<l \leqslant$ $m$. Then, since $o\left(y_{l}\right)=\alpha_{l}>\alpha_{k}, y_{l} \notin O_{\alpha_{k}} \supset N\left(i_{k}, x\right)$. Since $\{N(i, x): i \in \omega\}$ is a decreasing sequence of sets, this implies $i_{k}>i_{l}$. Therefore $0 \leqslant i_{m}<i_{m-1}<\cdots<i_{0}$, so $i_{0} \geqslant m$. But then $y_{0} \in N\left(i_{0}, x\right) \subset N(m, x) \subset O_{o(x)}$, in contradiction with $o\left(y_{0}\right)$ $=\alpha_{0}>o(x)$. This proves that $\mathcal{O}^{\prime}$ is point-finite.

EXAmple 1.4. Let $D^{*}=D \cup\{y\}$ be the one-point compactification of an uncountable discrete space by a point $y$. For every $m \in \omega$ and $x \in D^{*}$, let

$$
N(m, x)= \begin{cases}\{x, y\} & \text { if } m=0 \\ \{x\} & \text { otherwise }\end{cases}
$$


Then $\{N(m, x): m \in \omega, x \in X\}$ is clearly a point-network for $D^{*}$.

EXAMPLE 1.5. Take an ordinal $\lambda \geqslant 1$, and consider the set $X=\{f: f$ is a function; $\operatorname{dom}(f)$ is an ordinal $<\omega$; $\operatorname{range}(f) \subset \lambda\}$. Define a topology on $X$ in the following way:

If $\operatorname{dom}(f)=n \in \omega$, then all sets of the form $u(f, H)=U_{f}-U_{h \in H} U_{h}$, where $U_{f}=\{g \in X: g \uparrow n=f\}$ and $H$ is a finite subset of $U_{f}$, constitute a clopen neighbourhood base for $f$.

$X$ with this topology will be a zero-dimensional $T_{2}$ topology with character $\lambda$. Further, we can define a point-network for $X$ by putting $N(m, f)=\{f\} \cup\{f \uparrow k$ : $m \leqslant k \leqslant \operatorname{dom}(f)\}$ for every $f \in X$ and $m \in \omega$.

EXAMPLE 1.6. Example 9.1 of [Ce] is an example of a stratifiable, nonmetrizable space. It can easily be shown that this space has a point-network $\{N(m, x): m \in \omega$, $x \in X\}$ such that each $N(m, x)$ is a neighbourhood of $x$. (This observation is due to P. J. Collins and A. W. Roscoe.)

2. Stratifiability. The following lemma is the heart of our paper.

LemMa 2.1. Let $X$ be a $T_{1}$ space with a point-network. Then $X$ is the union of $a$ $\sigma$-discrete subspace and a subspace with a $G_{\delta}$-diagonal. Moreover, if $\psi(X)=\omega$, then $X$ has a $G_{\delta}$-diagonal.

Proof. It is enough to prove that $X^{2}-\Delta=\cup_{m \in \omega} M_{m}$ in such a way that if $m \in w$ and $K_{m}=\left\{x \in X:\langle x, x\rangle \in \bar{M}_{m}\right\}$, then

$$
K_{m}^{(m)}=\varnothing \text {. }
$$

To prove this, let us fix a point-network $\{N(m, x): m \in \omega, x \in X\}$ for $X$. Further, for every $m \in \omega$, let

$$
M_{m}=\left\{\langle x, y\rangle \in X^{2}-\Delta: N(m, x) \cap N(m, y)=\varnothing\right\} .
$$

Clearly, $\cup_{m \in \omega} M_{m}=X^{2}-\Delta$, so fix $m \in \omega$, and let us prove (*). Suppose indirectly that there is a point $z \in K_{m}^{(m)}$. Then we can inductively define sequences $\left\{z_{0}, \ldots, z_{m}\right\}$ of points and $\left\{B_{0}, \ldots, B_{m}\right\}$ of open sets of $X$ in such a way that $z_{0}=z, B_{0}=X$, and for $k=0, \ldots, m-1$

$$
z_{k+1} \in K_{m}^{(m-k-1)}
$$

and

$$
z_{k+1} \in B_{k+1} \subset V\left[z_{k}, B_{k}\right]-\left\{z_{0}, \ldots, z_{k}\right\} .
$$

To see that this can be done note that by $z_{k} \in K_{m}^{(m-k)}$ there is a point

$$
z_{k+1} \in K_{m}^{(m-k-1)} \cap\left(V\left[z_{k}, B_{k}\right]-\left\{z_{0}, \ldots, z_{k}\right\}\right) \quad\left(\subset B_{k}\right) .
$$

(Indeed, if there was no such point $z_{k+1}$, then the open set $U=V\left[z_{k}, B_{k}\right]-$ $\left\{z_{0}, \ldots, z_{k-1}\right\}$ would isolate $z_{k}$ in $K_{m}^{(m-k-1)}$ in contradiction with $z_{k} \in K_{m}^{(m-k)}$.) Then the choice $B_{k+1}=V\left[z_{k}, B_{k}\right]-\left\{z_{0}, \ldots, z_{k}\right\}$ will do.

Having done this, let us consider $V=V\left[z_{m}, B_{m}\right]$. Then, since $z_{m} \in K_{m}$ (and thus, $\left.\left\langle z_{m}, z_{m}\right\rangle \in \bar{M}_{m}\right)$ there is a pair $\langle x, y\rangle \in(V \times V) \cap M_{m}$. Since for $k=0, \ldots, m$ we have $x, y \in V\left[z_{k}, B_{k}\right]$, for each $k=0, \ldots, m$ there are $i_{k}, j_{k} \in \omega$ with

$$
z_{k} \in N\left(i_{k}, x\right) \subset B_{k} \text { and } z_{k} \in N\left(j_{k}, y\right) \subset B_{k} .
$$


By (2), if $k<l \leqslant m$, then $z_{k} \notin N\left(i_{l}, x\right)\left(\subset B_{l}\right)$. Since $\{N(i, x): i \in \omega\}$ is decreasing, this implies $i_{k}<i_{l}$. Thus $0 \leqslant i_{0}<i_{1}<\cdots<i_{m}$. Consequently, $i_{m} \geqslant m$. A similar argument proves $j_{m} \geqslant m$. Since $\langle x, y\rangle \in M_{m}, N\left(i_{m}, x\right) \cap N\left(j_{m}, y\right) \subset$ $N(m, x) \cap N(m, y)=\varnothing$. On the other hand, $z_{m} \in N\left(i_{m}, x\right) \cap N\left(j_{m}, y\right)$. This contradiction proves $K_{m}^{(m)}=\varnothing$.

Now, suppose $\psi(X)=\omega$. To prove that $X$ has a $G_{\delta}$-diagonal in this case, it is enough to see that each set $K_{m}^{*}=\bar{M}_{m} \cap \Delta=\left\{\langle x, x\rangle: x \in K_{m}\right\}$ is a $G_{\delta}$ set in $X^{2}$. Since by $(*), K_{m}^{*}$ is the union of $\leqslant m$ discrete subspaces, and the union of finitely many $G_{\delta}$ subsets is a $G_{\delta}$ subset, we only have to prove that every discrete subspace $D^{*}=\{\langle d, d\rangle: d \in D\}$ of $\Delta$ is a $G_{\delta}$ subset of $X^{2}$. Since $X$ is hereditarily collectionwise normal and $D$ is a discrete subspace of $X$, there is a disjoint open collection $\left\{O_{d}: d \in D\right\}$ in $X$ separating the points of $D$. Then it is easy to see that $\left\{O_{d} \times O_{d}\right.$ : $d \in D\}$ is a disjoint open separation (in $X^{2}$ ) of the points of $D^{*}$. Since $\psi(X)=\omega$ implies $\psi\left(X^{2}\right)=\omega$, all the points of $D^{*}$ are $G_{\delta}$ subsets in $X^{2}$. Thus $D^{*}$ is a $G_{\delta}$ subset of $X$.

Remark. As is shown by Example 1.5, one cannot improve Lemma 2.1 in many ways. One cannot prove, for example, that every space with a point-network is the union of a $\sigma$-closed discrete subspace and a subspace with a $G_{\delta}$-diagonal.

In what follows we shall apply the following well-known characterization of stratifiable spaces:

(*) $X$ is stratifiable iff it has a system $\{A(n, x): n \in \omega, x \in X\}$ of open sets such that

(1) $x \in A(n, x)$ for every $n \in \omega$ and $x \in X$, and

(2) given a closed subset $F$ of $X$ and a point $x \notin F$, there is an $n \in \omega$ with $x$ $\notin \overline{\cup\{A(n, y): y \in F\}}$.

TheORem 2.2 (MAIN). Let $X$ be a $T_{1}$ space with a point-network. Then $X$ is the union of a $\sigma$-discrete and a stratifiable subspace. Moreover, if $\psi(X)=\omega$, then $X$ is stratifiable.

Proof. By Lemma 2.1, it is enough to prove that if $X$ has a point-network $\{N(m, x): m \in \omega, x \in X\}$ and a $G_{\delta}$-diagonal sequence of open covers $\left\{\mathscr{G}_{n}: n \in \omega\right\}$, then $X$ is stratifiable.

We claim that if for every point $x \in X$ and every $n \in \omega$, we choose an element $G(n, x)$ of $\mathscr{G}_{n}$ containing $x$ and put $A(n, x)=V[x, V[x, G(n, x)]]$, then this assignment saiisfies properties (1) and (2) in the characterization of a stratifiable space.

(1) is clear. In order to show (2), let $x, F$ be given. Let $m \in \omega$ be the first, such that $N(m, x) \cap F=\varnothing$, and choose $n \in \omega$ so big that $N(i, x) \not \subset \operatorname{st}\left(x, \mathscr{G}_{n}\right)$ for every $i<m$. Then this $n \in \omega$ will do.

To see this, first we show $x \notin \bigcup\{V[y, G(n, y)]: y \in F\}$. Indeed, if there were a $y \in F$ with $x \in V[y, G(n, y)]$, then there would be an $i \in \omega$ such that $y \in N(i, x)$ $\subset G(n, y) \subset \operatorname{st}\left(x, \mathscr{G}_{n}\right)$. Since $N(i, x) \not \subset \operatorname{st}\left(x, \mathscr{G}_{n}\right)$ for $i<m$, it follows that $i \geqslant m$. But we choose $m$ so that $N(m, x) \cap F=\varnothing$, in contradiction with $y \in N(i, x) \subset$ $N(m, x)$. 
Next we show that $V[x, X-F] \cap \bigcup\{A(n, y): y \in F\}=\varnothing$, thereby proving $x \notin \overline{\cup\{A(n, y): y \in F\}}$. This follows since if we had $V[x, X-F] \cap A(n, y)=$ $V[x, X-F] \cap V[y, V[y, G(n, y)]] \neq \varnothing$ for some $y \in F$, then by Proposition 1.1 either $y \in X-F$ or $x \in V[y, G(n, y)]$ would hold, the first of which cannot hold by $y \in F$, and the second which cannot hold by the previous paragraph.

Corollary 2.3 (G. M. ReEd [R]). A $T_{\text {: }}$ space is metrizable if and only if it has an open point-network.

Proof. Let $\{N(m, x): m \in \omega, x \in X\}$ be an open point-network for $X$, and take a system $\{A(n, x): n \in \omega, x \in X\}$ satisfying (1) and (2) in the characterization of stratifiable spaces. For every $n \in \omega$, let $\mathscr{B}_{n}$ be a locally finite open refinement of the open cover $\mathscr{A}_{n}=\{A(n, x): x \in X\}$ of $X$. For every $B \in \mathscr{B}_{n}$, let us fix a point $x(n, B)$ of $X$ with $B \subset A(n, x(n, B))$, and define

$$
\mathscr{B}_{n m}=\left\{B_{n} N(m, x(n, B)): B \in \mathscr{B}_{n}\right\} .
$$

Clearly, $\mathscr{B}=\bigcup\left\{\mathscr{B}_{n m}: n, m \in \omega\right\}$ is a $\sigma$-locally finite open family in $X$. We are going to show that $\mathscr{B}$ also is a base for $X$.

To see this, let $U$ be an open set of $X$, and let $x \in U$. For every $n \in \omega$, choose a $B_{n} \in \mathscr{B}_{n}$ such that $x \in B_{n}$. Then $x \in A\left(n, x\left(n, B_{n}\right)\right)$ for every $n \in \omega$, so by property (2) in the characterization of stratifiable spaces, there is an $n \in \omega$ with $x\left(n, B_{n}\right) \in V[x, U]$. Fix this $n \in \omega$. By the definition of a point-network there is an $m \in \omega$ with $x \in N\left(m, x\left(n, B_{n}\right)\right) \subset U$. Thus $B=B_{n} \cap N\left(m, x\left(n, B_{n}\right)\right) \in \mathscr{B}_{n m} \subset \mathscr{B}$ and $x \in B \subset U$. Q.E.D.

ACKNOWLEDGEMENT. Between the submission and revision of this paper the author received a preprint of $\left[\mathbf{C R}_{1}\right]$ and other news from Collins. It turns out from these that our historical remarks should be corrected on two points.

1. Lemma 1.3 was (in an even more general form) independently proved by the authors of $\left[\mathbf{C R}_{1}\right]$.

2. What we called "Reed's theorem" was independently proved by M. E. Rudin, P. J. Collins and A. W. Roscoe. Thus it should well bear the names of all four authors of $\left[\mathbf{C R}_{\mathbf{1}}\right]$.

\section{REFERENCES}

[Ce] J. G. Ceder, Some generalizations of metric spaces, Pacific J. Math. 11 (1961), 105-125.

[C $\mathbf{C}_{1}$ ] P. J. Collins, Talk given at ICM 82, Warsaw, August 1983.

$\left[\mathrm{C}_{2}\right]$, Letter to the author, September 1983.

[CR $\left.\mathbf{C R}_{\mathbf{1}}\right]$ P. J. Collins, G. M. Reed, A. W. Roscoe and M. E. Rudin, A lattice of conditions on topological spaces, preprint.

[CR $\left.\mathbf{C R}_{2}\right]$ P. J. Collins and A. W. Roscoe, Criteria for metrizability, Proc. Amer. Math. Soc. 90 (1984), 631-640.

[E] R. Engelking, General topologv, PWN, Warsaw, 1977.

[R] G. M. Reed, Handwritten notes, October 1983.

Department of Mathematics, Kossuth University, Debrecen, Hungary H - 4010 